\title{
Chaotic immune symbiotic organisms search for SVC installation in voltage security control
}

\author{
Mohamad Khairuzzaman Mohamad Zamani, Ismail Musirin, Saiful Izwan Suliman, \\ Muhammad Murtadha Othman \\ Power System Operation Computational Intelligence (POSC) Research Group, \\ Centre for Electrical Power Engineering Studies (CEPES), Faculty of Electrical Engineering, \\ Universiti Teknologi MARA, Malaysia
}

\begin{abstract}
Article Info
Article history:

Received Jan 21, 2019

Revised April 23, 2019

Accepted May 25, 2019

\section{Keywords:}

Chaotic immune symbiotic organisms search (CISOS)

Fast voltage stability index (FVSI)

Static VAr compensator (SVC)

Total voltage deviation index (TVDI)

ABSTRACT

Parallel with the urbanization of the world, energy demand in the world also increased. The increase in energy demand will require a power system to be operated near its stability limit. To mitigate the problem, Flexible Alternating Current Transmission System (FACTS) devices can be installed as a compensation scheme to improve voltage security in a power system. For an effective compensation, FACTS devices should be optimally allocated in a power system. Although optimization techniques can be implemented to optimally allocate these devices, problems have been reported which would affect the performance of the optimization techniques in terms of producing high quality solutions. This paper presents the implementation of Chaotic Immune Symbiotic Organisms Search for solving optimal Static VAr Compensator (SVC) allocation problem for voltage security control. The optimization is validated in IEEE 26-Bus Reliability Test System (RTS) realizes the capability of CISOS in solving the optimization problem. Comparative studies with respect to Particle Swarm Optimization (PSO) and Evolutionary Programming (EP) resulting in good agreement on the results and demonstrated superior performance of CISOS. Results of the study can be beneficial to power system community in terms of compensation planning prior to real world implementation.
\end{abstract}

Copyright $\odot 2019$ Institute of Advanced Engineering and Science. All rights reserved.

\section{Corresponding Author:}

Mohamad Khairuzzaman Mohamad Zamani,

Power System Operation Computational Intelligence (POSC) Research Group,

Centre for Electrical Power Engineering Studies (CEPES), Faculty of Electrical Engineering,

Universiti Teknologi MARA, Malaysia.

Email: mohd_khairuzzaman@yahoo.com

\section{INTRODUCTION}

In recent years, ever increasing energy demand has increase the stress on power system which eventually would cause a power system to operate near its stability limit $[1,2]$. In order to mitigate this situation, several scheme can be implemented to improve the stability of a power system such as installation of power generation unit [3], reconfiguration of power networks [4] as well as installation of compensation devices.

Flexible AC Transmission System (FACTS) devices can be installed to provide compensation to power system. Static Var Compensator (SVC) is a type of shunt-connected FACTS devices which is capable of injecting and drawing reactive power from or to a power system. Due to this flexibility, SVC is preferred over capacitor bank in providing reactive power support to a power system [5]. However, optimal installation of SVCs are important for an effective compensation. To achieve this optimality, optimization techniques are implemented to determine the optimal location and sizing of SVCs to be installed in a power system. Various studies have been conducted to optimally allocate FACTS devices in power systems to solve various 
issues related to power system operations. Authors in [6] has determined the optimal allocation of SVC on IEEE 30-Bus using Genetic Algorithm (GA). Similar optimization technique is also implemented by [7] to determine the optimal allocation of SVC on a 13-Bus power system with the aim of improving voltage profile, minimize power loss and minimize investment cost. In [8], Raj and Bhattacharyya has implemented Whale Optimization Algorithm (WOA) to optimally allocate SVC and TCSC in IEEE 30-Bus and IEEE 57Bus systems to solve reactive power planning problem. An attempt to minimize active power loss and improve voltage profile of 57-bus test system via optimal allocation of shunt capacitor, SVC and STATCOM has been conducted by Sirjani et al. [9] by using Novel Global Harmony Search (NGHS) algorithm. Bhattacharyya and Gupta [10] has solve reactive power optimization problem using fuzzy-based evolutionary algorithms in which that the location of the FACTS devices are determined using Fuzzy Logic while the evolutionary algorithms will determine the optimal settings for the power system. The evolutionary algorithms used in [10] are GA and Differential Evolution (DE). A modified version of Gravitational Search Algorithm (GSA) known as Cumulative Gravitational Search Algorithm (CGSA) has been proposed to solve optimal STATCOM allocation problem in power system [11].

Although metaheuristics-based optimization techniques are able to solve optimal FACTS device allocation problem, problems have been reported related to the performance of the optimization technique such as dependency to parameter of optimization technique, tendency to stuck in local optima, slow convergence time and high computational burden, which eventually prevent the optimization technique from producing high quality solutions [5, 12]. Hence, a higher-performance optimization technique is needed so that the FACTS device can be optimally installed in a power system which would provide effective compensation. Symbiotic Organisms Search (SOS) optimization technique was first developed by Cheng \& Prayogo [13] in 2014 which was inspired by the symbiosis relationship of organisms in the ecosystem. The performance of SOS has been acknowledged by several studies conducted. In previous studies, SOS has been implemented to solve optimal SVC allocation for voltage profile improvement [5], economic dispatch [14, 15], optimal power flow [16] as well as civil engineering design [17]. Further modifications are proposed on SOS to improve its performance. Tejani et al. [18] has introduced adaptive element on the benefit factor of the SOS. Abdullahi et al. [19] has substituted the uniformly-distributed random number on the mutualism and commensalism updating expression by using random number generated by using chaos sequence. Saha et al. [20] has introduced chaotic local search (CLS) algorithm before the convergence test which would attempt to find a better solution around the current best solution. This methodology has been implemented and validated by Mohamad Zamani et al. [21].

This paper presents the implementation of Chaotic Immune Symbiotic Organisms Search (CISOS) optimization technique to solve optimal SVC allocation for voltage security control. In this paper, the location and sizing of the SVC will be determined by CISOS. The optimization problem will be demonstrated on IEEE 26-Bus Reliability Test System (RTS). Comparative studies are also conducted with SOS, Particle Swarm Optimization (PSO) and Evolutionary Programming (EP) highlights the superiority of CISOS in solving the optimal allocation problem in terms of solution quality produced by the optimization techniques.

\section{RESEARCH METHODOLOGY}

In order to solve the optimal allocation of SVC problem for voltage security control, CISOS technique is applied to determine the optimal location and sizing of SVC to be installed in the test system to improve the voltage security of the system.

\subsection{Problem Formulation}

The first objective of the study is to improve the voltage profile of the system through the minimization of total voltage deviation index (TVDI). The fitness function for the first optimization objective $f_{l}$ as well as mathematical representation of TVDI are expressed as in (1).

$$
f_{1}=T V D I=\sum_{i=1}^{N_{b}}\left(\frac{V_{r e f, i}-V_{i}}{V_{r e f, i}}\right)^{2}
$$

where $V_{\text {ref }, i}$ is the reference value of $i^{\text {th }}$ bus voltage, $V_{i}$ is the $i^{\text {th }}$ bus voltage and $N_{b}$ is the number of buses in the test system. In [5], TVDI is calculated only for load buses. For this paper, the calculation of TVDI will include all buses in the system. The voltage reference value for load buses are set to 1.00 p.u. while the reference value for slack and $\mathrm{P}-\mathrm{V}$ buses are set with their voltage setpoint value as in the power flow solution algorithm [21]. 
The second objective of the study is to improve the voltage stability of the system. The voltage stability of the system is evaluated by using Fast Voltage Stability Index (FVSI) developed by Musirin et al. [22]. Voltage stability of the system is represented by the highest FVSI value in the entire test system. FVSI is mathematically expressed as in (2) while the fitness function of the second optimization objective $f_{2}$ is expressed as in (2).

$$
\begin{aligned}
& F V S I_{a b}=\frac{4 Z_{a b}^{2} Q_{b}}{V_{a}^{2} X_{a b}} \\
& f_{2}=F V S I_{\text {max }}=\max \left(F V S I_{a b}\right)
\end{aligned}
$$

where $F V S I_{\max }$ is the highest $F V S I$ value from the entire test system and $F V S I_{a b}$ is the $F V S I$ value for the transmission line connecting $a^{\text {th }}$ bus and $b^{\text {th }}$ bus in the test system. The sending bus and receiving bus of the transmission lines are represented by $a^{\text {th }}$ bus and $b^{\text {th }}$ bus respectively. Voltage value of the sending bus is represented by $V_{a}$ and the reactive power flowing into the receiving bus is represented by $Q_{b}$. The reactance and impedance of the transmission line connecting $a^{\text {th }}$ bus to $b^{\text {th }}$ bus are represented by $X_{a b}$ and $Z_{a b}$ respectively.

Normally, optimization techniques are used to solve single-objective optimization problem. However, this study requires the proposed optimization technique to solve the optimization problem considering both objectives. In order to achieve the goal, multi-objective optimization mode should be implemented. In this paper, weighted sum method is applied to solve multi-objective optimization problem by combining all fitness functions and weighted by using weighing factor [23]. Hence, the objective function of the optimization is expressed as:

$$
\begin{aligned}
& \text { O.F. } \operatorname{minimize}(f) \\
& f=\left(w_{1} \times f_{1}\right)+\left(w_{2} \times f_{2}\right)
\end{aligned}
$$

where $f$ is the overall fitness value which is the sum of the weighted fitness values and $O . F$. is the objective function. The weighing factor for the first and second fitness values are represented by $w_{1}$ and $w_{2}$.

\subsection{Constraints}

In weighted sum method, sum of the weighing factors used in (5) should be equal to 1 [24]. This constraint can be expressed as in (6) where $w_{i}$ is the weighing factor of $i^{\text {th }}$ fitness function and $N_{o b j}$ is the number of optimization objectives.

$$
\sum_{i=1}^{N_{o b j}} w_{i}=1
$$

It is possible for an SVC to inject or draw reactive to or from a bus in a power system. In this paper, the SVC is modelled as a negative reactive power load. Positive SVC sizing value $Q_{s v c}$ indicates the reactive power from the SVC into the bus. On the other hand, negative value of $Q_{s v c}$ implies the value of reactive power drawn from the bus into the SVC. The sizing of SVC is limited by its minimum and maximum capacity of the SVC indicated by $Q_{s v c}^{\min }$ and $Q_{s v c}^{\max }$ respectively. This constraint can be expressed as:

$$
Q_{s v c}^{\min } \leq Q_{s v c} \leq Q_{s v c}^{\max }
$$

In this paper, SVC is proposed to be installed at load buses since slack bus and P-V bus have the capability to regulate reactive power. Load bus is defined as a bus with a load connected to it. A bus with no load or power generation unit connected to it is known as connecting bus, and SVC is not proposed to be installed there. The constraint of location of SVC installation can be expressed as:

$$
1 \leq L O C_{s v c} \leq N_{l b}
$$

where $N_{l b}$ is the number of load buses in the system.

\subsection{Chaotic Immune Symbiotic Organisms Search (CISOS) for Optimal SVC Installation}

To solve the optimal SVC allocation problem, the authors have proposed the implementation of CISOS optimization technique to determine the optimal location and sizing of SVC for voltage security control in power system. CISOS is first developed by Mohamad Zamani et al. [25] which is inspired by the 
inclusion of diversification and improved exploration capability through the introduction of element of cloning, elimination and Chaotic Local Search (CLS) to the original Symbiotic Organisms Search (SOS) optimization technique developed by Cheng and Prayogo [13]. Detailed information about CISOS can be referred in [25], and the process of CISOS in solving optimal SVC allocation problem for voltage security control can be summarized as follows:

\subsubsection{Step 1: Initialization}

Firstly, the optimization parameters are set which are number of initial organisms in the ecosystem $N_{i o}$, Number of clones $N_{\text {clone }}$, iteration counter iter, maximum number of iteration max_iter, and CLS control parameter $p$. Then, a set of possible solution known as the organism is generated by using random number. A set of organism is made up of 2 parts, namely SVC sizing and the location of SVC. The SVC sizing is generated using random number bounded with the limits specified as in (7) while the SVC location is generated using integer random number bounded by limits specified as in (8).

$$
\begin{gathered}
\text { iter }=1 \\
Q_{s v c(i, d)}=\operatorname{rand}\left(Q_{s v c}^{\min }, Q_{s v c}^{\max }\right) \\
\operatorname{LOC}_{s v c(i, d)}=\operatorname{rand} \operatorname{Int}\left(1, N_{l b}\right) \\
X_{\operatorname{init}}=\left\{\begin{array}{c}
Q_{s v c(1,1)}, \ldots, Q_{s v c(1, d)}, \cdots, Q_{s v c\left(1, N_{s v c}\right)}, \operatorname{LOC}_{s v c(1,1)}, \cdots L O C_{s v c(1, d)}, \cdots, L O C_{s v c\left(1, N_{s v c}\right)} \\
Q_{s v c(i, 1)}, \ldots, Q_{s v c(i, d)}, \cdots, Q_{s v c\left(i, N_{s v c}\right)}, L C_{s v c(i, 1)}, \cdots L O C_{s v c(i, d)}, \cdots, L O C_{s v c\left(i, N_{s v c}\right)} \\
\vdots \\
Q_{s v c\left(N_{i o}, 1\right)}, \ldots, Q_{s v c\left(N_{i o}, d\right)}, \cdots, Q_{s v c\left(N_{i o}, N_{s v c}\right)}, L O C_{s v c\left(N_{i o}, 1\right)}, \cdots L O C_{s v c\left(N_{i o}, d\right)}, \cdots, L O C_{s v c\left(N_{i o}, N_{s v c}\right)}
\end{array}\right\}
\end{gathered}
$$

while $Q_{s v(i, d)}$ is the sizing of $d^{\text {th }}$ unit SVC of $i^{\text {th }}$ initial organism, $L O C_{s v c(i, d)}$ is the location of $d^{\text {th }}$ unit SVC of $i^{\text {th }}$ initial organism, $N_{i o}$ is the number of initial organisms in the ecosystem and $N_{s v c}$ is the number of SVC to be installed. In (10), $\operatorname{rand}()$ is the function to generate random number and $\operatorname{randInt()}$ in (11), is the function to generate integer random number.

After the ecosystem has been occupied by the initial organisms, then the fitness value of the initial organisms is computed. Then, an organism with the best fitness is chosen to be initialized as the best organism. Lastly, the chaotic search space radius is computed by using (13), which consists of (14) and (15).

$$
\begin{aligned}
& r=\left\{r_{1(1,1)}, \cdots r_{1(1, d)}, \cdots r_{1\left(1, N_{s v c}\right)}, r_{2(1,1)}, \cdots, r_{2(1, d)}, \cdots r_{2\left(1, N_{s v c}\right)}\right\} \\
& r_{1(1, d)}=\frac{Q_{s v c(d)}^{\max }-Q_{s v c(d)}^{\min }}{2} \\
& r_{2(1, d)}=\frac{L O C_{s v c(d)}^{\max }-L O C_{s v c(d)}^{\min }}{2}
\end{aligned}
$$

\subsubsection{Step 2: Cloning}

Size of the ecosystem is increased by cloning the initial organisms. The initial organisms will be duplicated by a factor of $N_{\text {clone }}$. After the cloning process, the organisms $X$ will be subjected to the next optimization step. The act of cloning to produce $X$ can be expressed as:

$$
X=\left[\begin{array}{c}
X_{\text {init }}^{(1)} \\
X_{\text {init }}^{(2)} \\
\vdots \\
X_{\text {init }}^{\left(N_{\text {clone }}\right)}
\end{array}\right]
$$

After cloning, the number of organisms in the ecosystem has increased by a multiple of $N_{\text {clone }}$, and the total number of organisms in the ecosystem after cloning $N_{o T}$ can be computed as:

$$
N_{o T}=N_{\text {io }} \times N_{\text {clone }}
$$



value of 1 .

After the initial organisms have been duplicated, then the organism counter $i$ is initialized to

$$
i=1
$$

\subsubsection{Step 3: Symbiosis Relationship Phases}

The organisms are subjected to symbiosis relationship phases consisting of mutualism, commensalism and parasitism. Firstly, an organism known as the $i^{\text {th }}$ organism $X_{i}$ is chosen from the ecosystem. Then, another organism known as $j^{\text {th }}$ organism $X_{j}$ is randomly chosen from the same ecosystem. When choosing $X_{j}$, $j^{\text {th }}$ organism should not be the same organism as the $i^{\text {th }}$ organism. Then, both organisms are subjected to mutualism phase, which then produce new organisms represented by $X_{i, \text { new }}$ and $X_{j, \text { new }}$. $X_{i, \text { new }}$ and $X_{j, \text { new }}$ are produced by

$$
\begin{aligned}
& M V=\frac{X_{i}+X_{j}}{2} \\
& B F 1=\operatorname{rand} I n t(1,2) \\
& B F 2=\operatorname{randInt}(1,2) \\
& X_{i, \text { new }}=X_{i}+\operatorname{rand}(0,1) \times\left(X_{\text {best }}-M V \times B F 1\right) \\
& X_{j, \text { new }}=X_{j}+\operatorname{rand}(0,1) \times\left(X_{\text {best }}-M V \times B F 2\right)
\end{aligned}
$$

where $M V$ is the mutual vector computed by (19), $X_{\text {best }}$ is the best organism in the ecosystem, $B F 1$ and $B F 2$ are benefit factors, $\operatorname{rand}(0,1)$ is a random number ranged between 0 to 1 , and $\operatorname{randInt}(1,2)$ is an integer random number between 1 to 2 . It should be noted that location of SVC in $X_{i, \text { new }}$ and $X_{j, \text { new }}$ produced from the updating process should be rounded off to the nearest integer.

Then, the fitness values of $X_{i, \text { new }}$ and $X_{j, \text { new }}$ are computed and compared with the fitness values of $X_{i}$ and $X_{j}$ respectively. If the fitness value of $X_{i, \text { new }}$ is lower than $X_{i}$, then $X_{i, \text { new }}$ will replace $X_{i}$ in the ecosystem. The same case also applies to $X_{j, \text { new }}$ and $X_{j}$.

Next, the organisms are subjected to commensalism phase. Maintaining the same $X_{i}$, another random organism is chosen from the ecosystem to be $X_{j}$ in which that $X_{j}$ is not the same organism as $X_{i}$. Then, a new organism $X_{i, n e w}$ is produced by subjecting $X_{i}$ and $X_{j}$ to commensal relationship which is expressed as

$$
X_{i, \text { new }}=X_{i}+\operatorname{rand}(-1,1) \times\left(X_{\text {best }}-X_{j}\right)
$$

where $\operatorname{rand}(-1,1)$ is a random number between -1 to 1 . The location of SVC in $X_{i, \text { new }}$ produced by (24) should be rounded off to the nearest integer. The fitness value of $X_{i, n e w}$ is computed and compared with the fitness value of $X_{i}$. Should $X_{i \text {,new }}$ possess a lower fitness value compared to $X_{i}$, then $X_{i, \text { new }}$ will replace $X_{i}$ in the ecosystem.

Final phase in symbiosis relationship phases is parasitism phase. The same $X_{i}$ is maintained, an organism $X_{j}$ is randomly chosen from the ecosystem. The same rule for choosing $X_{j}$ in mutualism and commensalism also applies to choosing $X_{j}$ in parasitism. Then a parasite $P V$ is produced by duplicating $X_{i}$. Then random dimension of $P V$ is modified by using random number as in (10) for SVC sizing and (11) for SVC location. Then, fitness value of $P V$ is computed and compared to fitness value of $X_{j}$. Should the fitness value of $P V$ be lower than $X_{j}$, then $P V$ will replace $X_{j}$ in the ecosystem.

\subsubsection{Step 4: Best Organism Identification}

A candidate for best organism is chosen among the organisms in the ecosystem in which that the organism possesses the lowest fitness value. Then the fitness value of the candidate is compared to the fitness value of current best organism. If the best organism candidate has a lower fitness value compared to the fitness value of current best organism, then the best organism candidate will replace the current best organism as the best organism. Otherwise, the current best organism is retained.

\subsubsection{Step 5: Symbiosis Relationship Termination Test}

The optimization algorithm will check either all the organisms has undergone the symbiosis relationship phases. If the organism counter $i$ has reached total number of organisms after cloning $N_{o T}$, 
the optimization process will continue to step 6. Otherwise, the organism counter is increased by 1 , and the optimization process continues to step 3.

\subsubsection{Step 6: Elimination}

The size ecosystem is reduced from $N_{o T}$ organisms to $N_{i o}$. To reduce the ecosystem size, CISOS will choose $N_{i o}$ fittest organisms among the entire organisms in the ecosystem. This way, only the fitter organisms will be carried forward to next iteration.

\subsubsection{Step 7: Chaotic Local Search (CLS)}

A new organism is generated around the best organism using CLS. Firstly, CLS iteration counter $c h$ and chaotic variable $c v$ are initialized. The number of dimensions of the chaotic variable should be equal to the number of dimensions of $X_{\text {best }}$.

$$
\begin{aligned}
& c h=0 \\
& c v_{(c h, c)}=\operatorname{rand}(0,1)
\end{aligned}
$$

where $c$ is the dimension of $c v$.

Then, chaotic variable is updated by using Piecewise Linear Chaotic Map (PLCM).

$$
c v_{(c h+1, c)}=\left\{\begin{array}{cc}
\frac{c v_{(c h, c)}}{p} & c v_{(c h, c)} \in(0, p) \\
\frac{\left(1-c v_{(c h, c)}\right)}{(1-p)} & c v_{(c h, c)} \in[p, 1)
\end{array}\right.
$$

where $\mathrm{p}$ is the control parameter of CLS.

After the chaotic variable is updated, a new organism is produced by wrapping the chaotic variable around the best organism $X_{\text {best }}$ using (28). It should be noted that the location of SVC produced by (28) should be rounded off to the nearest integer.

$$
X_{c l s(c h+1, c)}=X_{\text {best }(1, c)}+r_{(1, c)}\left(2 c v_{(c h+1, c)}-1\right)
$$

The fitness value of the produced organism is computed and compared with the fitness value of the best organism. If the fitness value of the new organism is higher than the fitness value of $X_{\text {best }}$, then the CLS iteration counter is increased by 1 and the CLS algorithm proceeds to chaotic variable update.

$$
c h=c h+1
$$

In the case of new organism having lower fitness value compared to $X_{\text {best }}$, then the new organism will replace $X_{\text {best }}$, CLS algorithm is terminated and the chaotic search space radius is reduced by using (30). In $(30), \operatorname{rand}(0,1)$ is a random number ranged from 0 to 1 .

$$
r_{(1, c)}=r_{(1, c)}+\operatorname{rand}(0,1)
$$

\subsubsection{Step 8: Convergence Test}

In this step, CISOS will determine whether the algorithm should continue or stop. If the value of iter has reached the maximum CISOS iteration number max_iter, then the optimization process will stop, and $X_{\text {best }}$ will be the optimal solution produced by CISOS. Otherwise, value of iter will be incremented by 1 , and the optimization process continues to step 2.

\section{RESULTS AND DISCUSSION}

In this paper, CISOS is implemented to solve optimal SVC allocation for voltage security control problem in power system. The test system used in the study is IEEE 26-Bus Reliability Test System (RTS). IEEE 26-Bus RTS consists of 17 load buses and 6 power generation units. In the optimization process, 3 units of SVC will be installed in which that the location and sizing of the SVC will be determined using CISOS. The parameters of the optimization process are listed in Table 1. 
Table 1. Optimization parameter of CISOS

\begin{tabular}{cc}
\hline Optimization parameter & Parameter value \\
\hline Number of generated organisms & 20 \\
Number of clones & 10 \\
Maximum iteration of CISOS algorithm & 200 \\
CLS control parameter & 0.4 \\
Maximum CLS iteration & 100 \\
\hline
\end{tabular}

The optimization process will run for 20 times for each case study to observe the variation of optimal solutions produced by CISOS. The results which would be analysed is the fitness value of the optimal solutions which are made up of TVDI and FVSI $I_{\text {max }}$ values. The effectiveness of CISOS in solving the allocation problem is demonstrated through comparative studies with respect to Particle Swarm Optimization (PSO) and Evolutionary Programming (EP) techniques.

\subsection{Base Case Condition}

During this condition, the test system experience no change from its nominal condition. Optimization techniques are implemented to solve the optimal SVC allocation problem on IEEE 26-Bus RTS. The best optimal solutions produced by CISOS, PSO and EP are tabulated as in Table 2.

Table 2. Results of Voltage Security Improvement Using SVC During Base Case Condition

\begin{tabular}{cccccc}
\hline \multirow{2}{*}{ Technique } & SVC Sizing (MVAr) & SVC Location (Bus No.) & TVDI & FVSI $I_{\max }$ & Fitness Value \\
\hline Pre-optimized & - & - & 0.00483 & 0.35376 & 0.17929 \\
CISOS & $60.2433,40.9218,37.5464$ & $9,12,11$ & 0.00463 & 0.33607 & 0.17035 \\
PSO & $100.0000,-76.6738,100.0000$ & $6,9,22$ & 0.00162 & 0.34324 & 0.17243 \\
EP & $-0.8570,71.2966,-86.8921$ & $21,20,18$ & 0.00292 & 0.34294 & 0.17293 \\
\hline
\end{tabular}

From the results obtained, it can be observed that the optimization techniques are able to solve the optimal SVC allocation problem. All techniques have produced optimal solutions with lower fitness values compared to the pre-optimized fitness value. The results also revealed that CISOS has managed to record the best optimal solution which having the lowest fitness value compared to the optimal solutions produced by PSO and EP. The optimal solution produced by CISOS has improved the voltage profile of the system indicated by the reduction of TVDI value from 0.00483 to 0.00463 . However, the optimal solution produced by CISOS provides maximum improvement to the voltage stability of the system which is indicated by $5 \%$ reduction of $F V S I_{\max }$ value from 0.35376 down to 0.33607 . The optimal solution produced by EP provides better voltage profile improvement, but it only manages to produce lesser improvement on voltage stability of the system. Although the optimal solution produced by PSO provides maximum improvement on the voltage profile of the system, the improvement on the voltage stability is minimal compared to CISOS and EP.

\section{CONCLUSION}

This paper has presented the implementation of Chaotic Immune Symbiotic Organisms Search (CISOS) optimization technique to solve optimal SVC allocation problem on IEEE 26-Bus RTS for voltage security control. It is desirable for a power system to have a good voltage security since a good voltage security suggests a power system to be healthy and able to maintain stable operation. Implementation of optimization techniques can help to achieve this while mitigating overcompensation and under-compensation issues. While the fitness value has been reduced through the implementation of optimal solutions produced, the superiority of performance of CISOS compared to PSO and EP has been proved in terms of the quality of the optimal solution produced.

\section{ACKNOWLEDGEMENTS}

The authors would like to acknowledge The Institute of Research Management and Innovation (IRMI) UiTM Shah Alam, Selangor, Malaysia for the financial support of this research. This research is supported by IRMI under the Fundamental Research Grant Scheme (FRGS) with project code: 600-RMI/FRGS 5/3 (0102/2016). 


\section{REFERENCES}

[1] P. Wang, et al., "Power Generation Expansion Optimization Model Considering Multi-Scenario Electricity Demand Constraints: A Case Study of Zhejiang Province, China," Energies, vol. 11, pp. 15, 2018.

[2] A. Aghahosseini, et al., "A Techno-Economic Study of an Entirely Renewable Energy-Based Power Supply for North America for 2030 Conditions," Energies, vol. 10, pp. 28, 2017.

[3] L. G. Meegahapola, et al., "Voltage Security Constrained Reactive Power Optimization Incorporating Wind Generation," in 2012 IEEE International Conference on Power System Technology (POWERCON), pp. 1-6, 2012.

[4] A. Onlam, et al., "Power Loss Minimization and Voltage Stability Improvement in Electrical Distribution System via Network Reconfiguration and Distribution Generation Placement Using Novel Adaptive Shuffled Frogs Leaping Algorithm," Energies, vol. 12, pp. 12, 2019.

[5] M. K. M. Zamani, et al., "Symbiotic Organisms Search Technique for SVC Installation in Voltage Control," Indonesian Journal of Electrical Engineering and Computer Science, vol. 6, pp. 318-329, May 2017.

[6] S. Dixit, et al., "Optimal placement of SVC for minimizing power loss and improving voltage profile using GA," in 2014 International Conference on Issues and Challenges in Intelligent Computing Techniques (ICICT), pp. 123129, 2014.

[7] I. Pisica, et al., "Optimal SVC placement in electric power systems using a Genetic Algorithms based method," in 2009 IEEE Bucharest PowerTech, pp. 1-6, 2009.

[8] S. Raj and B. Bhattacharyya, "Optimal placement of TCSC and SVC for reactive power planning using Whale optimization algorithm," Swarm and Evolutionary Computation, vol. 40, pp. 131-143, Dec 2018.

[9] R. Sirjani, et al., "Optimal allocation of shunt Var compensators in power systems using a novel global harmony search algorithm," International Journal of Electrical Power and Energy Systems, vol. 43, pp. 562-572, Dec 2012.

[10] B. Bhattacharyya and V. K. Gupta, "Fuzzy based evolutionary algorithm for reactive power optimization with FACTS devices," International Journal of Electrical Power \& Energy Systems, vol. 61, pp. 39-47, Oct 2014.

[11] M. Packiasudha, et al., "A new Cumulative Gravitational Search algorithm for optimal placement of FACT device to minimize system loss in the deregulated electrical power environment," International Journal of Electrical Power \& Energy Systems, vol. 84, pp. 34-46, Jan 2017.

[12] R. Dubey, et al., "Optimal placement of shunt FACTS devices using heuristic optimization techniques: An overview," in 2014 Fourth International Conference on Communication Systems and Network Technologies, pp. 518-523, 2014.

[13] M. Y. Cheng and D. Prayogo, "Symbiotic Organisms Search: A new metaheuristic optimization algorithm," Computers \& Structures, vol. 139, pp. 98-112, Jul 2014.

[14] D. Das, et al., "Symbiotic organisms search algorithm for economic dispatch problems," in 2017 Second International Conference on Electrical, Computer and Communication Technologies (ICECCT), pp. 1-7, 2017.

[15] D. C. Secui, "Large-scale multi-area economic/emission dispatch based on a new symbiotic organisms search algorithm," Energy Conversion and Management, vol. 154, pp. 203-223, Dec 2017.

[16] D. Prasad and V. Mukherjee, "A novel symbiotic organisms search algorithm for optimal power flow of power system with FACTS devices," Engineering Science and Technology, an International Journal, vol. 19, pp. 79-89, Mar 2016.

[17] D. Prayogo, et al., "A Novel Implementation of Nature-inspired Optimization for Civil Engineering: A Comparative Study of Symbiotic Organisms Search," Civil Engineering Dimension, vol. 19, pp. 36-43, Mar 2017.

[18] G. G. Tejani, et al., "Adaptive symbiotic organisms search (SOS) algorithm for structural design optimization," Journal of Computational Design and Engineering, vol. 3, pp. 226-249, Jul 2016.

[19] M. Abdullahi, et al., "Chaotic symbiotic organisms search for task scheduling optimization on cloud computing environment," in 2017 6th ICT International Student Project Conference (ICT-ISPC), pp. 1-4, 2017.

[20] S. Saha and V. Mukherjee, "Optimal placement and sizing of DGs in RDS using chaos embedded SOS algorithm," IET Generation, Transmission \& Distribution, vol. 10, pp. 3671-3680, Nov 2016.

[21] M. K. M. Zamani, et al., "Chaos Embedded Symbiotic Organisms Search Technique for Optimal FACTS Device Allocation for Voltage Profile and Security Improvement," Indonesian Journal of Electrical Engineering and Computer Science, vol. 8, pp. 146-153, Oct 2017.

[22] I. Musirin and T. K. A. Rahman, "Novel fast voltage stability index (FVSI) for voltage stability analysis in power transmission system," in Student Conference on Research and Development, pp. 265-268, 2002.

[23] S. Ranganathan, et al., "Self-adaptive firefly algorithm based multi-objectives for multi-type FACTS placement," IET Generation, Transmission \& Distribution, vol. 10, pp. 2576-2584, Aug 2016.

[24] E. E. Hassan, et al., "Multi objective adaptive tumbling bacterial foraging in VAR solutions for sustainable power system operation," Indonesian Journal of Electrical Engineering and Computer Science, vol. 11, pp. 129-136, Jul 2018.

[25] M. K. M. Zamani, et al., "Chaotic Immune Symbiotic Organisms Search for solving optimisation problem," International Journal of Engineering \& Technology, vol. 7, pp. 73-79, Aug 2018. 